\title{
Kariesprophylaxe in der kieferorthopädischen Praxis: Entwicklung einer medizinischen Leitlinie*
}

\section{Caries Preventive Measures in Orthodontic Practice: The Develop- ment of a Clinical Practice Guideline}

Autoren

Barbara C. M. Oosterkamp¹, Wil J. M van der Sanden², Jo E. F. M. Frencken³, Anne Marie Kuijpers-Jagtman'

Institute

1 Department of Orthodontics and Craniofacial Biology, Radboud University Medical Center, Nijmegen, Niederlande

2 Department of Global Oral Health, Radboud University Medical Center, Nijmegen, Niederlande

3 Department of Preventive and Restorative Dentistry, Radboud University Medical Center, Nijmegen, Niederlande

\section{Schlüsselwörter}

Delphi, evidenzbasierte Praxis, White-Spot-Läsionen, Prophylaxemaßnahmen, Demineralisation

Key words

Delphi, evidence-based practice, white spot lesions, preventive measures, tooth demineralization

Bibliografie

DOI https://doi.org/10.1055/s-0043-112086

Inf Orthod Kieferorthop 2017; 49: 122-131

(c) Georg Thieme Verlag KG Stuttgart · New York ISSN 0020-0336

Korrespondenzadresse

Dr. B.C.M. Oosterkamp

Radboud University Medical Center

PO Box 9101

6500 HB Nijmegen

Niederlande

orthodontics@dent.umcn.nl

\section{ZUSAMMENFASSUNG}

Zielsetzung Initialkaries oder White-Spot-Läsionen (WSL) sind eine Begleiterscheinung kieferorthopädischer Behandlungen. Sie führen zu ästhetischen Problemen und beinhalten das Risiko für tiefergehende Schmelz- und Dentinläsionen. Zur Prophylaxe der WSL sind viele Strategien entwickelt worden, die angewandten Präventionsmaßnahmen sind im Detail jedoch sehr unterschiedlich. In der vorliegenden Studie werden Maßnahmen zusammengestellt, mit deren Hilfe eine medizinische
Leitlinie entwickelt werden kann. Diese ermöglicht es dem Kieferorthopäden, Prophylaxemaßnahmen auf Grundlage der besten verfügbaren Evidenz auszuwählen.

Material und Methoden Zur Anwendung kam ein nominales Gruppenverfahren (RAND-e-modifiziertes Delphi-Verfahren). Eine multidisziplinär zusammengesetzte Expertenkommission beurteilte insgesamt 264 praxis- und evidenzbasierte Maßnahmen zur Prophylaxe der WSL. Um sicherzustellen, dass die Kommissionsmitglieder auf demselben Wissensstand waren, sollten die Mitglieder als Vorbereitung auf die 3 Konsensrunden insgesamt 6 Publikationen durcharbeiten, die über eine systematische Literaturauswertung zusammengestellt worden waren. Gemäß dem Verfahren wurde eine Übereinstimmung von mehr als $75 \%$ innerhalb jedes einzelnen der 3 Punkte umfassenden Abschnitte der 9-Punkte-Skala als Konsens angesehen. Ergebnisse Nach der ersten und der zweiten Konsensrunde war Konsens bei 37,5 bzw. bei 31,1\% der Maßnahmen erreicht worden. Bei den verbliebenen 31,4\% der Maßnahmen wurde Konsens während einer 4 Stunden dauernden Konferenz erzielt. Schlussfolgerungen Aus einer systematischen Literaturauswertung gewonnene Maßnahmen zur Prophylaxe der WSL wurden mit der Einschätzung von Experten kombiniert und mithilfe eines nominalen Gruppenverfahrens formal zu einem Konsens zusammengefasst. Diese Maßnahmen bildeten die Grundlage für die Formulierung einer Leitlinie zur Prophylaxe der WSL vor und während einer kieferorthopädischen Behandlung.

\section{ABSTRACT}

Objectives White spot lesions (WSLs) are a side effect of orthodontic treatment, causing esthetic problems and a risk of deeper enamel and dentine lesions. Many strategies have been developed for preventing WSLs, but great variability exists in preventive measures between orthodontists. This study developed statements on which a clinical practice guideline (CPG) can be developed in order to help orthodontists select preventive measures based on the best available evidence.

Materials and methods A nominal group technique (RAND-e modified Delphi procedure) was used. A multidisciplinary expert panel rated 264 practice- and evidence-based statements rela-

* Dieser Artikel ist im englischen Original erschienen in: Orthod Craniofac Res 
ted to the management of WSLs. To provide panel members with the same knowledge, a total of 6 articles obtained from a systematic review of the literature were read by the panel in preparation of 3 consensus rounds. According to the technique, a threshold of $75 \%$ of all ratings within any 3-point section of the 9-point scale regarding a specific statement was accepted as consensus.

Results After the first and second consensus rounds, consensus was reached on 37.5 and $31.1 \%$ of statements, respectively. For the re- maining $31.4 \%$ of statements, consensus was reached during a 4 -h consensus meeting.

Conclusions Statements on the management of WSLs derived from a systematic literature review combined with expert opinion were formally integrated toward consensus through a nominal group technique. These statements formed the basis for developing a CPG on the management of WSLs before and during orthodontic treatment.

\section{Einführung}

Bei fast $25 \%$ der festsitzend behandelten Patienten sind nach Abschluss der Therapie Demineralisationen des Zahnschmelzes erkennbar [1]. Aufgrund ihres Aussehens werden solche Stellen in der kieferorthopädischen Literatur häufig als Kreideflecken (Initialkaries, White-Spot-Läsionen) bezeichnet. Nach der Entbänderung bleiben diese Entkalkungen oft permanent bestehen, was zu ästhetischen Problemen führt und das Risiko erhöht, dass sich der Prozess fortsetzt [1, 2]. Eine Untersuchung unter dänischen Kieferorthopäden ergab deutliche Unterschiede bei den durchgeführten Präventionsmaßnahmen und ließ erkennen, dass publizierte wissenschaftliche Erkenntnisse in der Praxis oft wenig Beachtung finden. In der gleichen Studie sprachen sich 68\% der Teilnehmer für die Entwicklung einer medizinischen Leitlinie für die Prophylaxe der WSL aus [3]. In einer solchen Leitlinie werden Erkenntnisse aus der Literatur und klinische Erfahrung zusammengeführt. Sie kann damit zu einer stärker evidenzorientierten Tätigkeit in der täglichen Praxis beitragen. Durch Implementierung einer medizinischen Leitlinie lassen sich die Häufigkeit von WSL verringern und die Unterschiede zwischen den angewandten Verfahren zur Prophylaxe der Initialkaries beseitigen.

Die verfügbare Literatur ist sich hinsichtlich der Prophylaxe der WSL jedoch nicht einig, was häufig auf ein mangelhaftes Studiendesign zurückzuführen ist [4,5]. Die Entwicklung einer klinischen Leitlinie zu dieser Thematik kann daher nur gelingen, wenn Ergebnisse aus wissenschaftlich fundierter Forschung und klinisches $\mathrm{Er}$ fahrungswissen in einer so genannten Konsensrichtlinie zusammengeführt werden. Zentrale Aspekte für die Akzeptanz besonders von konsensbasierten Leitlinien in der täglichen Praxis sind Vertrauen in deren Qualität und die Glaubwürdigkeit ihrer Entwickler [6]. Eine der Voraussetzungen dafür ist daher die Verwendung eines methodisch anerkannten Verfahrens zur Konsensfindung unter den Entwicklern.

Ziel der vorliegenden Studie war die Entwicklung von Aussagen zur Kariesprophylaxe während einer kieferorthopädischen Behandlung mit festsitzenden Apparaturen mithilfe eines methodisch sicheren Verfahrens zur Konsensfindung. Diese Aussagen bilden dann die Grundlage für die Formulierung einer medizinischen Leitlinie für Kieferorthopäden und Allgemeinzahnärzte.

\section{Methoden}

\section{Studiendesign}

Der Konsensbildungsprozess wurde mithilfe eines RAND-e-modifizierten Delphi-Verfahrens durchgeführt. Bei einem Delphi-Verfahren werden Einschätzungen zu einer bestimmten Fragestellung mit Fragebögen gesammelt [7]. Grundlage ist dabei die Prämisse, dass sich Einzelentscheidungen durch gepooltes Wissen, die kollektive Einschätzung einer Expertengruppe, verbessern. Die RANDe-modifizierte Delphi-Methode ist besonders gut zur Konsensfindung bei Fragestellungen geeignet, für die in der Fachliteratur nicht genügend Nachweise für eine optimale Therapie vorhanden sind. Dazu wird eine Gruppe von Experten zusammengestellt, die einzeln ihre Meinung zur Zweckmäßigkeit von verschiedenen Behandlungsstrategien bei einer Vielzahl unterschiedlicher klinischer Fälle äußern. Die einzelnen Fälle werden charakteristischerweise auf Karteikarten präsentiert.

\section{Konsensfindung nach dem RAND-e-modifizierten Delphi-Verfahren}

Um potenzielle evidenzbasierte Strategien zur Kariesprophylaxe zu finden und um die Mitglieder der Expertenkommission auf denselben Wissensstand zu bringen, wurde eine systematische Literaturrecherche durchgeführt und die Ergebnisse nach veröffentlichten Richtlinien auf ihre Evidenz geprüft. Die Einträge zwischen Mitte 2002 und 2008 in den Datenbanken PubMed, Medline und Cochrane wurden systematisch nach relevanten Veröffentlichungen durchsucht. Für die Jahre vor 2002 lag bereits eine systematische Auswertung der Literatur vor [4]. Die Recherche erbrachte insgesamt 6 Studien [4, 8-12]. Die Charakteristika dieser Untersuchungen zeigt $>$ Tab. 1. Die Literatursuche wurde zu einem späteren Zeitpunkt bis 2014 ausgedehnt, wobei eine Cochrane-Studie gefunden wurde [5]. Diese Auswertung ergab eine mittlere Evidenz dafür, dass die Applikation von Fluoridlack alle 6 Wochen während

- Tab. 1 Übersicht über die 6 in der vorliegenden Studie verwendeten Publikationen nach dem zweiten Schritt des Suchverfahrens.

\begin{tabular}{|l|l|l|l|}
\hline Studie & $\begin{array}{l}\text { Erschei- } \\
\text { nungsjahr }\end{array}$ & Land & $\begin{array}{l}\text { Art der } \\
\text { Studie }\end{array}$ \\
\hline Benson et al. [9] & 2005 & Großbritannien & SR \\
\hline Benson et al. [10] & 2004 & Großbritannien & CSR \\
\hline Chadwic et al. [11] & 2005 & Großbritannien & SR \\
\hline Derks et al. [4] & 2004 & Niederlande & SR \\
\hline Zimmer u. Rottwinkel [12] & 2004 & Deutschland & RCT \\
\hline Stecksén-Blicks [8] & 2007 & Schweden & RCT \\
\hline
\end{tabular}

SR: systematische Übersicht, CSR: Cochrane systematische Übersicht, RCT: randomisierte kontrollierte Untersuchung 
einer kieferorthopädischen Behandlung kariesprophylaktisch wirkt. Diese Empfehlung wurde in die Leitlinie aufgenommen, da die entsprechende Studie vor 2008 veröffentlicht worden war. Sie wurde den Mitgliedern der Expertengruppe auch zur Lektüre vorgelegt [8].

\section{Entwicklung digitaler Fallkarten für kieferorthopädi- sche Patienten anhand des Risikos}

Die Patientenkarten wurden nach umfangreichen Gesprächen mit Kariologen entworfen. Dabei wurden alle relevanten und allgemein akzeptierten Risikofaktoren für die Entstehung von Karies berücksichtigt. Für jede Kombination aus Mundgesundheit, Hygienestatus und Ernährungsgewohnheiten wurde eine Patientenkarte angelegt. Jede dieser Karten zeigte 4 farbige Intraoralaufnahmen eines Patienten. Mundgesundheit, Mundhygiene (Plaque- und Blutungsindex) und Ernährungsgewohnheiten des jeweiligen Patienten wurden kurz beschrieben ( $\triangleright \mathbf{A} \mathbf{b} \mathbf{b} . \mathbf{1})$. Das eigens dafür entwickelte Computerprogramm, mit dem die insgesamt 23 Patientenkarten aufgerufen werden konnten ( $\triangleright$ Abb. 2 ), wurde vorab von 3 Kieferorthopäden und 4 Allgemeinzahnärzten getestet und, wo erforderlich, nachgebessert. Die Patienten wurden neben den bereits beschriebenen Kariesrisiken als medizinisch gesund eingestuft (American Society of Anesthesiologists (ASA) Stufe 1). Sie hatten denselben sozioökonomischen Status, eine (stabile) Zusammensetzung des Speichels und waren angehalten worden, die Zähne 2-mal täglich mit einer hoch fluoridhaltigen Zahnpasta (1450 ppm Fluorid) zu putzen. Die festsitzenden Apparaturen sollten noch mind. ein weiteres Jahr lang getragen werden. Für jedes Mitglied der Expertengruppe wurde ein Speicher-Stick vorbereitet, auf dem sich neben den Patientenkarten mit den klinischen Informationen in Form von Tabellen, Abbildungen und Bildern ein Programm befand, mit dem die Karten aufgerufen werden konnten.

\section{Entwicklung der Empfehlungen}

Neben der Beschreibung des jeweiligen Falles enthielt jede der Patientenkarten 10 Empfehlungen zu Prophylaxemaßnahmen vor Beginn der kieferorthopädischen Behandlung und 2 weitere für mögliche Maßnahmen im Verlauf der Therapie. Diese Empfehlungen umfassten Anweisungen zur Mundhygiene und die Aufforderung, eine elektrische Zahnbürste zu verwenden, die Verwendung von Chlorhexidinlösung und -gel, zusätzliche Fluoridapplikationen sowie eine Ernährungsberatung ( $\triangleright$ Abb. 1). Die Empfehlungen wurden auf Grundlage der evidenzbasierten kariesprophylaktischen Maßnahmen formuliert, die im Rahmen der systematischen Literaturauswertung gefunden worden waren. Zusätzlich wurden die evidenzbasierten Entscheidungshilfen zur Kariesprophylaxe der Dutch National Association for the Promotion of Oral Hygiene herangezogen, der nationalen niederländischen Organisation für die Schulung in Mundgesundheit (Ivoren Kruis).

\section{Auswahl und Vorbereitung der multidisziplinären Expertenkommission}

Die Expertenkommission wurde nach ihrer professionellen Qualifikation, der beruflichen Erfahrung und einem Wohnort in den Niederlanden zusammengestellt. Insgesamt wurden 11 Experten, allesamt ausgebildete Zahnärzte, nach den Empfehlungen der AGREE-Collaboration für die Entwicklung von evidenzbasierten medizinischen Leitlinien [13] aus den Bereichen Forschung, Lehre, Kieferorthopädie, Kariologie, allgemeine Zahnheilkunde und Gutachter von Versicherungen ausgewählt. Die Anzahl der Kommissionsmitglieder entsprach den Vorgaben für ein RAND-e-modifiziertes Verfahren [14]. Aus praktischen Gründen waren in dieser Phase des Prozesses noch keine Patientenvertreter beteiligt. Wir beschlossen, die Patientenperspektive bei dieser Leitlinie im Rahmen einer Implementationsstudie zu berücksichtigen, die in einem späteren Schritt durchgeführt werden soll. Dabei werden die Patienten gebeten, einen Fragebogen zu ihrer Akzeptanz der Empfehlungen auszufüllen.

Um sicherzustellen, dass alle Kommissionsmitglieder auf demselben Wissensstand waren, wurde die ausgewählte Literatur ( $\triangleright$ Tab. 1) mit der Bitte, diese durchzuarbeiten, zusammen mit weiteren Informationen zur Kariesprophylaxe und dem Zweck der Untersuchung vorab an sämtliche Experten verschickt. Die Mitglieder der Kommission wurden freundlich gebeten, sämtliches Material sorgfältig zu studieren.

\section{Erste Runde der Konsensfindung nach dem RAND-e- modifizierten Delphi-Verfahren}

3 Wochen nachdem den Kommissionsmitgliedern die Literatur zugesandt worden war, erhielten sie den Speicher-Stick mit den Patientenkarten. Diese enthielten Bilder und klinische Informationen über die jeweiligen Patienten. Außerdem wurden Informationen darüber mitgeteilt, wie die Patientenkarten zu beurteilen waren. Die Experten gründeten ihre Einschätzungen zur Zweckmäßigkeit der vorgeschlagenen Maßnahmen sowohl auf deren Vorteile (Wirksamkeit und Effektivität) als auch auf die Nachteile (Risiken und Kosten). Die Einschätzungen wurden mithilfe der RAND-Skala quantifiziert, die von Stufe 1 (ich halte die Behandlung für absolut unzweckmäßig) bis Stufe 9 (ich halte die Behandlung für absolut zweckmäßig) reichte. Für die Auswertung sollte der Speicher-Stick innerhalb von 4 Wochen in einem frankierten Freiumschlag zurückgeschickt werden.

\section{Zweite Runde der Konsensfindung nach dem RAND- e-modifizierten Delphi-Verfahren}

Die Ergebnisse der ersten Bewertungsrunde wurden 3 Wochen später an jedes Kommissionsmitglied verschickt. Jeder der Experten konnte seine eigenen Bewertungen im Vergleich zum Medianwert aus sämtlichen Bewertungen erkennen. Die Teilnehmer sollten nun die Patientenkarten bei Bewertungen, bei denen sich kein Konsens eingestellt hatte, erneut beurteilen und ihre frühere Einschätzung gegebenenfalls revidieren. Die Speicher-Sticks mit den Neubewertungen sollten innerhalb von 2 Wochen wieder zurückgeschickt werden.

\section{Konsenskonferenz}

Als Vorbereitung für die Konsenskonferenz wurden auf Grundlage der Ergebnisse aus den beiden Bewertungsrunden allgemeine Aussagen zur Kariesprophylaxe in der kieferorthopädischen Praxis vorformuliert. Die Konferenz hatte zum Ziel, diejenigen Bewertungen zu diskutieren, über die auch nach der zweiten Bewertungsrunde kein Konsens erreicht worden war. Die Kommissionsmitglieder diskutierten über ihre unterschiedlichen Ansichten. Den Vorsitz führte dabei ein erfahrender Diskussionsleiter (A.K.), die Dauer der ein- 


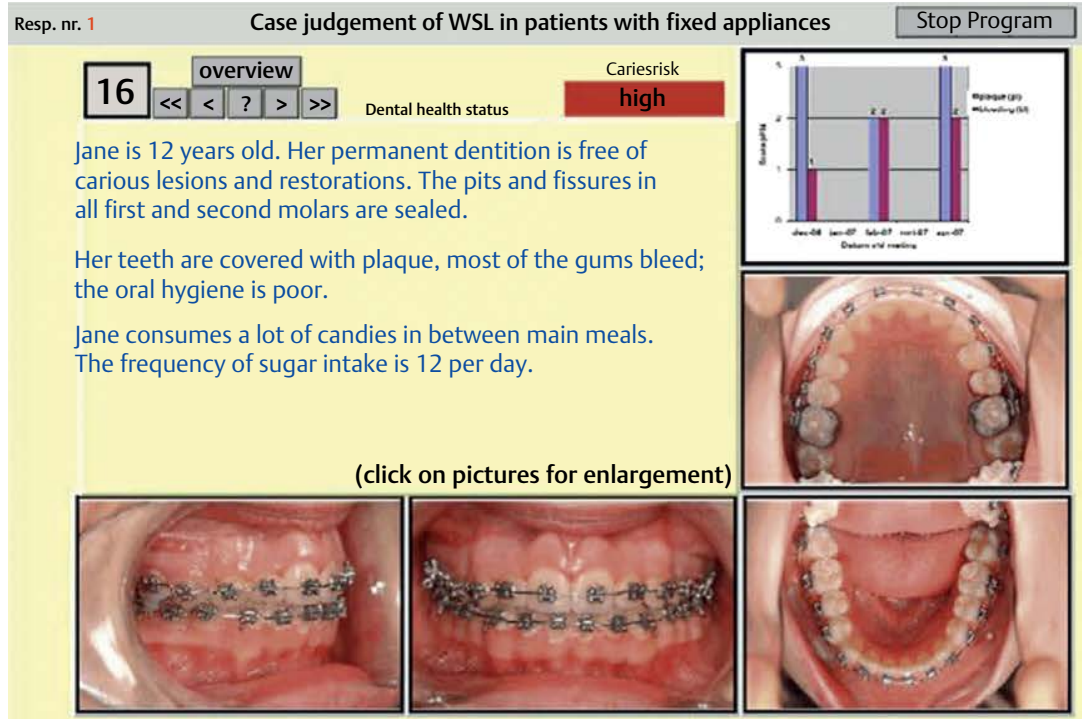

You are required first, to read the selected literature and then answer the statements presented below. for which you can use your clinical experience.

All patients are considered healthy (ASA-score 1), have the same social economic status, the same (stable) saliva composition and clean their teeth twice a day with fluoride containing tooth paste (1 $450 \mathrm{ppm}$ fluoride).

Each statement requires a score on a 9-point scale, which ranges from 1 [I totally disagree] to 9 [I totally agree]

1. Patient should receive oral hygiene instruction choice itd 010203040506070809 ita

2. Patient should brush with an electronically powered tooth brush itd 010203040506070809 ita

3. Patient and parents should receive clarification on the oral health situation itd 010203040506070809 ita

4. Patient and parents should receive a warning letter itd 010203040506070809 ita

5. Patient should rinse with a chlorhexidine mouth rinse itd 010203040506070809 ita

6. Patient should receive a chlorhexidine application itd 010203040506070809 ita

7. Patient should brush with a high ppm fluoride containing tooth paste itd 010203040506070809 ita

8. Patient should rinse with a fluoride mouth rinse itd $010203040506 \circ 70809$ ita

9. Patient should receive a fluoride application itd 010203040506070809 ita

10. Patient should receive a tailor-made dietary advice itd 010203040506070809 ita

11. The orthodontic treatment should continue with removable appliances only itd 010203040506070809 ita

12. The fixed appliances should be removed itd 010203040506070809 ita 13. Other measures:

Abb. 1 Beispiel für eine kieferorthopädische Patientenkarte während einer Behandlung mit allen zu beurteilenden Maßnahmen. Itd: ich lehne die Maßnahme ab (I totally disagree); Ita: ich befürworte die Maßnahme (I totally agree). 

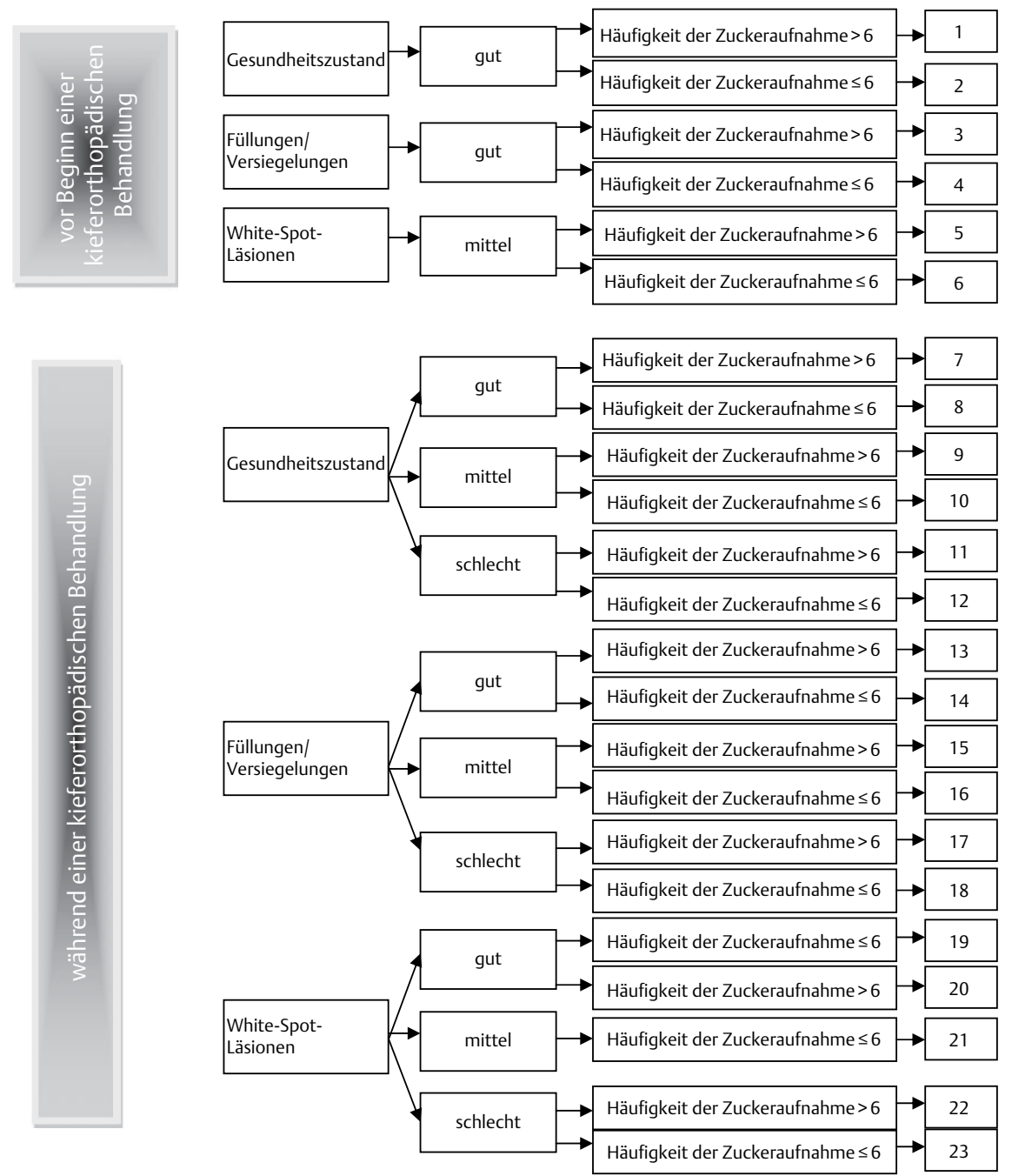

> Abb. 2 Die Entwicklung der Patientenkarte. Sämtliche Karten (die eine Gruppe von Patienten repräsentierten) waren als medizinisch gesund eingestuft worden [ASA-Wert 1], sie hatten denselben sozioökonomischen Status, dieselbe (stabile) Zusammensetzung des Speichels und putzten 2-mal täglich die Zähne mit einer hoch fluoridhaltigen Zahnpasta (1450 ppm Fluorid). Zahnstatus: gesund, Vorliegen oder Fehlen von (aktiven) kariösen Läsionen oder Füllungen. Mundhygienestatus: gut, mittelmäßig oder schlecht, abhängig von den Gingiva- und Plaquewerten. Ernährungsmuster: Zuckerzufuhr $>6$ oder $\leq 6$ mal pro Tag.

zelnen Redebeiträge wurde von 2 Personen notiert (WvdS und ID) und die Sitzung wurde aufgezeichnet. Am Ende der Diskussion waren sich sämtliche Teilnehmer über alle Patientenkarten einig und damit war die Konferenz beendet. Ein innerhalb einer Woche formuliertes abschließendes Konsenspapier wurde allen Kommissionsmitgliedern zur Zustimmung zugesandt.

\section{Datenanalyse}

Wie von der RAND-Collaboration empfohlen, wurde die Bewertungsskala in 3 Abschnitte unterteilt [14]. Ein Wert zwischen 1 und 3 bedeutete, dass die Experten die vorgeschlagene Maßnahme als nicht indiziert ansahen, bei 4-6 waren sie unentschieden und 7-9 zeigte an, dass sie die Prophylaxemaßnahme für angebracht hielten [15]. Nach dem RAND-e-modifizierten Delphi-Verfahren wurde eine Übereinstimmung von mehr als $75 \%$ innerhalb jedes einzelnen der 3 Punkte umfassenden Abschnitte als Konsens angesehen [14].
Nach der ersten und nach der zweiten Bewertungsrunde wurden für jede Bewertung die individuellen und die Gruppenmedianwerte sowie die prozentuale Übereinstimmung berechnet. Die Teilnehmer waren außerdem gebeten worden, die Zeit in Stunden zu notieren, die sie darauf verwandt hatten 1 ) die ausgewählte Literatur durchzuarbeiten, 2) die beiden Bewertungsdurchgänge durchzuführen und 3) zur Konsenskonferenz anzureisen. Für jedes Kommissionsmitglied wurden der gesamte und der durchschnittliche Zeitaufwand berechnet ( $\triangleright$ Tab. 2).

\section{Ergebnisse}

Jedes Kommissionsmitglied hatte insgesamt 264 Maßnahmen zu beurteilen (60 vor Behandlungsbeginn und 204 während der Therapie). In 3 Bewertungsrunden (RAND-e-modifiziertes Delphi-Verfahren Konsensrunde 1, RAND-e-modifiziertes Delphi-Verfahren 
- Tab. 2 Zeitaufwand der einzelnen Kommissionsmitglieder pro Studienabschnitt bei Durchlaufen des gesamten Konsensprozesses.

\begin{tabular}{|l|r|r|r|}
\hline & $\begin{array}{l}\text { Durchschnittli- } \\
\text { cher Zeitauf- } \\
\text { wand (h) }\end{array}$ & SD & $\begin{array}{l}\text { Streu- } \\
\text { breite (h) }\end{array}$ \\
\hline $\begin{array}{l}\text { Durcharbeiten der } \\
\text { ausgewählten Literatur }\end{array}$ & 3,5 & 1,9 & $2-7$ \\
\hline erste Beurteilung & 2,5 & 1,3 & $1,5-5$ \\
\hline zweite Beurteilung & 1,7 & 0,4 & $1-2$ \\
\hline Anreise zur Konsenskonferenz & 3,8 & 1,5 & $2-6$ \\
\hline Dauer der Konsenskonferenz & 4 & & \\
\hline $\begin{array}{l}\text { Durchschnittlicher Zeitauf- } \\
\text { wand pro Teilnehmer * }\end{array}$ & 15,5 & 2,9 & $12-19$ \\
\hline
\end{tabular}

* für einen Teilnehmer, der am gesamten Prozess teilgenommen hat

- Tab. 3 Anzahl der Maßnahmen in Patientenkarten vor Behandlungsbeginn $(n=6)$ bei Patienten mit geringem $(n=2)$, mittlerem $(n=2)$ und hohem $(n=2)$ Kariesrisiko.

\begin{tabular}{|l|l|l|l|l|}
\hline & $\begin{array}{l}\text { Runde } \\
\text { Maßnahme }\end{array}$ & $\begin{array}{l}\text { Runde } \\
\mathbf{2}\end{array}$ & $\begin{array}{l}\text { Diskus- } \\
\text { sion }\end{array}$ & $\begin{array}{l}\text { ge- } \\
\text { samt }\end{array}$ \\
\hline $\begin{array}{l}\text { Mundhygieneunterweisung } \\
\text { Verwendung einer }\end{array}$ & 6 & 0 & 0 & 6 \\
\hline $\begin{array}{l}\text { elektrischen Zahnbürste } \\
\text { Information der Eltern }\end{array}$ & 5 & 0 & 5 & 6 \\
\hline $\begin{array}{l}\text { schriftliche Information der } \\
\text { Eltern }\end{array}$ & 3 & 1 & 1 & 6 \\
\hline $\begin{array}{l}\text { Spülung mit Chlorhexidin } \\
\text { Applikation von Chlorhexidin }\end{array}$ & 5 & 0 & 1 & 6 \\
\hline $\begin{array}{l}\text { Putzen mit hoch fluoridhal- } \\
\text { tiger Zahnpasta }\end{array}$ & 1 & 1 & 4 & 6 \\
\hline $\begin{array}{l}\text { Spülung mit fluoridhaltiger } \\
\text { Lösung }\end{array}$ & 3 & 3 & 0 & 6 \\
\hline Applikation von Fluorid & 1 & 1 & 4 & 6 \\
\hline $\begin{array}{l}\text { individuelle Ernährungsbe- } \\
\text { ratung }\end{array}$ & 2 & 1 & 3 & 6 \\
\hline \begin{tabular}{l} 
gesamt \\
\hline
\end{tabular} & 32 & 8 & 20 & 60 \\
\hline
\end{tabular}

Konsensrunde 2 und Konsenskonferenz) wurde für alle Maßnahmen ein Konsens gefunden ( $\bullet$ Tab. 3 und $\ \mathbf{4}$ ). Der durchschnittliche Zeitaufwand betrug für jeden Teilnehmer 15,5 Stunden pro Runde ( $\triangleright$ Tab. 2).

Nach der ersten Bewertungsrunde bestand bei 32 der $60 \mathrm{Maß}$ nahmen vor Therapiebeginn und bei 67 der 204 Maßnahmen während der Behandlung Konsens (Gesamtkonsens 37,56\%; • Tab. 4 und $>5$ ). Die meisten Übereinstimmungen fanden sich bei den Mundhygieneunterweisungen (vor Behandlungsbeginn 6/6, während der Therapie 12/17) und bei der Beschreibung der aktuellen oralen Situation für die Eltern (vor Behandlungsbeginn 5/6, wäh-
- Tab. 4 Anzahl der Maßnahmen in Patientenkarten während einer Therapie $(n=17)$ bei Patienten mit geringem $(n=3)$, mittlerem $(n=7)$ und hohem $(n=7)$ Kariesrisiko.

\begin{tabular}{|l|l|l|l|l|}
\hline & $\begin{array}{l}\text { Runde } \\
\text { Maßnahme }\end{array}$ & $\begin{array}{l}\text { Runde } \\
\mathbf{2}\end{array}$ & $\begin{array}{l}\text { Diskus- } \\
\text { sion }\end{array}$ & $\begin{array}{l}\text { ge- } \\
\text { samt }\end{array}$ \\
\hline $\begin{array}{l}\text { Mundhygieneunterweisung } \\
\text { Verwendung einer elektrischen }\end{array}$ & 12 & 3 & 2 & 17 \\
\hline Zahnbürste & 0 & 14 & 3 & 17 \\
\hline $\begin{array}{l}\text { Information der Eltern } \\
\text { schriftliche Ermahnung der }\end{array}$ & 5 & 4 & 1 & 17 \\
\hline Eltern & 5 & 4 & 17 \\
\hline $\begin{array}{l}\text { Spülung mit Chlorhexidin } \\
\text { Applikation von Chlorhexidin }\end{array}$ & 3 & 3 & 11 & 17 \\
\hline $\begin{array}{l}\text { Putzen mit hoch fluoridhaltiger } \\
\text { Zahnpasta }\end{array}$ & 4 & 6 & 7 & 17 \\
\hline $\begin{array}{l}\text { Spülung mit fluoridhaltiger } \\
\text { Lösung }\end{array}$ & 3 & 12 & 2 & 17 \\
\hline $\begin{array}{l}\text { Applikation von Fluorid } \\
\text { individuelle Ernährungsberatung }\end{array}$ & 3 & 4 & 10 & 17 \\
\hline $\begin{array}{l}\text { Fortsetzung der festsitzenden } \\
\text { Behandlung }\end{array}$ & 8 & 5 & 5 & 17 \\
\hline $\begin{array}{l}\text { Abbruch der festsitzenden } \\
\text { Behandlung }\end{array}$ & 7 & 6 & 2 & 17 \\
\hline \begin{tabular}{l} 
gesamt \\
\hline
\end{tabular} & 67 & 74 & 63 & 204 \\
\hline
\end{tabular}

rend der Therapie 12/17). Nach der zweiten Bewertungsrunde war bei weiteren 82 (31,2\%) der verbliebenen 165 Maßnahmen Konsens erzielt worden ( $\bullet$ Tab. 3 und $\ \mathbf{4}$ ). Ein Kommissionsmitglied war ausgeschieden und nahm an der zweiten Runde nicht mehr teil.

In der Konsenskonferenz wurden insgesamt 83 Maßnahmen (20 vor Behandlungsbeginn, 63 während der Therapie) diskutiert, über die in den beiden ersten Runden kein Konsens erreicht werden konnte ( $\vee$ Tab. $\mathbf{3}$ und $\triangleright \mathbf{4}$ ). Bei den Patientenkarten vor Behandlungsbeginn betrafen die am meisten diskutierten Bewertungen die Verwendung einer elektrischen Zahnbürste (5/6) sowie die hoch fluoridhaltige Zahnpasta und die Fluoridapplikation (4/6). Bei den Patientenkarten während der Therapie wurde am meisten über die Fluoridapplikation (10/17), die Applikation von Chlorhexidin und die Mundspülung (12/17) sowie die Verwendung einer hoch fluoridhaltigen Zahnpasta (7/17) diskutiert. Nach insgesamt 4 Stunden war bei allen Maßnahmen Konsens erreicht worden. > Abb. 3 fasst das Konsensfindungsverfahren zusammen. Die daraus resultierende medizinische Leitlinie zeigen $>$ Abb. $\mathbf{4}$ und $\triangleright$ Tab. $\mathbf{5}$.

\section{Diskussion}

Die Kariesprophylaxe während einer kieferorthopädischen Behandlung wird nachgewiesenermaßen sehr unterschiedlich gehandhabt [3]. Wie in vielen Bereichen der Kieferorthopädie, lässt die verfügbare Literatur zu diesem Thema jedoch die methodische Stringenz vermissen, die für die Formulierung einer evidenzbasierten Richtlinie erforderlich wäre. Beim aktuellen Versuch wurde ein RAND-e-mo- 

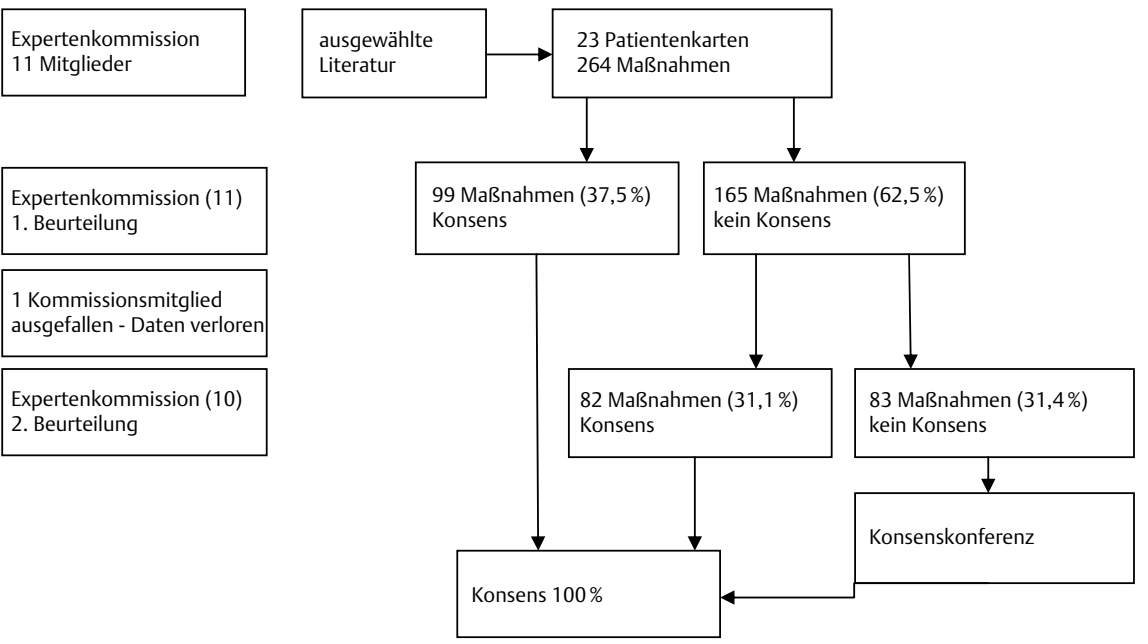

- Abb. 3 Schematischer Ablauf des in der vorliegenden Studie verwendeten RAND-e-modifizierten Delphi-Verfahrens mit absoluten und prozentualen Angaben zum Konsens.

difiziertes Delphi-Verfahren verwendet, um einen formalen Konsens zwischen Experten herbeizuführen. Bei diesem Verfahren kamen die Experten auf Grundlage ihrer Interpretation der Literatur, ihrer klinischen Erfahrung und in der direkten Diskussion während der Konsenskonferenz zu einer Einigung. Die Ergebnisse waren Grundlage für die Formulierung einer medizinischen Leitlinie zur Kariesprophylaxe, die als verlässliche Entscheidungshilfe für Kieferorthopäden und Allgemeinzahnärzte dienen kann. Dieses Verfahren ist bereits für die Erstellung von Konsenspapieren im Bereich der Mundhygiene angewandt worden. Unserer Kenntnis nach kommt es allerdings hier zum ersten Mal in der Kieferorthopädie zum Einsatz.

In der ersten Bewertungsrunde bestand Konsens in erster Linie bei Maßnahmen in Verbindung mit Mundhygieneinstruktionen. Dies überrascht nicht, da die Mundhygieneunterweisung als die wirkungsvollste und effektivste Maßnahme zur Kariesprophylaxe angesehen wird. Nach der zweiten Bewertungsrunde war bei weiteren 31,1\% der Maßnahmen Konsens erreicht worden. 31,4\% der Maßnahmen verblieben für die Diskussion im Rahmen der Konsenskonferenz. Die Diskussionen dieser Konferenz boten für alle Teilnehmer die Möglichkeit, ihr Wissen und ihre Erfahrung aus ihrem eigenen Fachgebiet einzubringen und von anderen bei bestimmten Themen zu lernen. Bei der Konsensfindung spielte das kollektive Wissen bei den diskutierten Maßnahmen eine große Rolle. Dies unterstreicht die Bedeutung einer multidisziplinär zusammengesetzten Kommission besonders in Fällen, bei denen Konsens über Maßnahmen erreicht werden soll, die nicht durch Evidenz aus der Literatur gestützt werden. Dies war der Fall bei den meisten Diskussionspunkten, insbesondere bei der Applikation von Chlorhexidin und der Mundspülung.

Die vorliegende Untersuchung wird dadurch begrenzt, dass die Formulierung der medizinischen Leitlinie mehrere Jahre gedauert hat. Die systematische Literaturauswertung, auf der die Leitlinie beruht, umfasste nur Veröffentlichungen bis 2008. Vielversprechende neuere Materialien zur Kariesprophylaxe, wie etwa APF-Schaum [16], Casein Phosphopeptid - amorphes Calciumphosphat (CPP-ACP) [17] und lichthärtende Versiegelungsmaterialien [18] werden bei der aktuellen Leitlinie nicht berücksichtigt. Die Literaturauswertung wurde inzwischen bis 2014 weitergeführt und erbrachte eine CochraneStudie [5]. In dieser Studie wurde die Aussage, die Applikation von fluoridhaltigem Lack alle 6 Wochen im Verlauf einer kieferorthopädischen Behandlung wäre eine wirkungsvolle Prophylaxemaßnahme, mit mittlerer Evidenz bewertet. Diese Empfehlung wurde in die Leitlinie aufgenommen, da die zugrunde liegende Studie vor 2008 veröffentlicht worden war. Die Untersuchung sollte auch von den Mitgliedern der Expertenkommission durchgearbeitet werden [8]. In Hinblick auf neue Verfahren zur Kariesprophylaxe möchten wir darauf hinweisen, dass die Entwicklung von Leitlinien ein kontinuierlicher Prozess ist. Die aktuelle Leitlinie wird vom Dutch Oral Health Guideline Institute verbreitet. Diese Institution richtet sich nach den Empfehlungen der AGREE-Collaboration [13]. Nach deren Empfehlungen wird die Leitlinie jährlich evaluiert und alle 3 Jahre wird eine neue Literaturauswertung durchgeführt. Anhand der Ergebnisse dieser neuen Auswertung entscheidet die Kommission für die Leitlinie darüber, ob ein neues Verfahren durchgeführt werden muss. Nach welchem Verfahren eine Aktualisierung durchgeführt wird, hängt dabei von der Art der Evidenz ab, die eingearbeitet werden muss. An einer solchen Aktualisierung werden klinische und methodologische Fachleute beteiligt.

Die vorliegende Leitlinie wird schrittweise in mehreren kieferorthopädischen Praxen implementiert, die in unser praxisorientiertes Forschungsnetzwerk eingebunden sind. Bei diesen Praxen wird die Anwendung der Leitlinie durch Extraktion der Daten über durchgeführte Prophylaxemaßnahmen und die damit verbundenen Kosten aus den elektronischen Patientenfiles überprüft. Außerdem wird die Häufigkeit von Initialkaries anhand von standardisierten vor und nach einer kieferorthopädischen Behandlung angefertigten Bildern beurteilt. Zusätzlich wird die Beurteilung der Leitlinie aus Sicht der Patienten und der Kieferorthopäden mithilfe von Fragebögen ermittelt. Das Protokoll der Studie wurde zur Veröffentlichung eingereicht. 


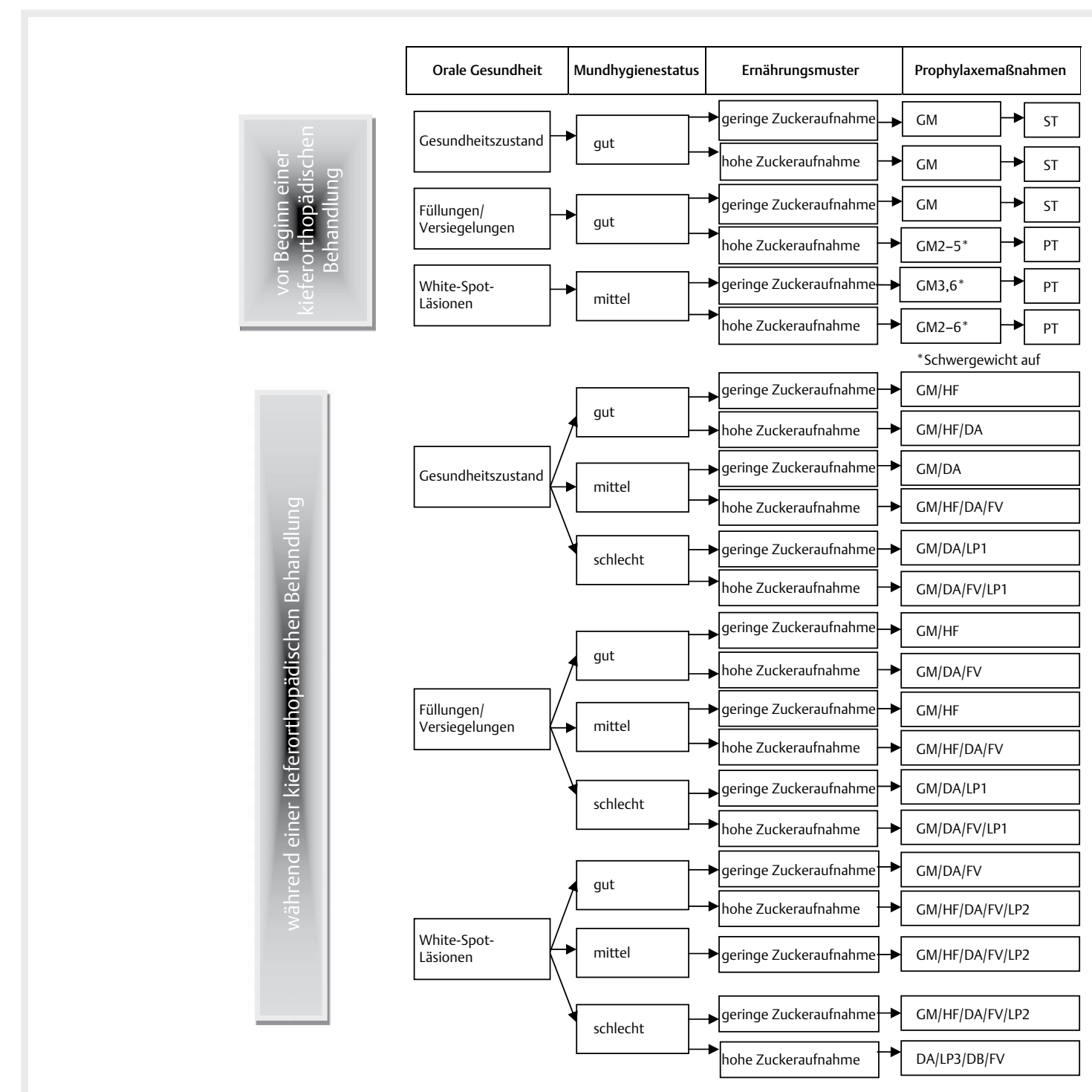

Abb. 4 Die endgültigen Prophylaxeanweisungen vor und während einer kieferorthopädischen Behandlung mit festsitzenden Apparaturen der medizinischen Leitlinie. Die Abkürzungen werden in $\triangleright$ Tab. 5 aufgeschlüsselt.

In der vorliegenden Studie wird ein einfaches und zuverlässiges Verfahren vorgestellt, mit dessen Hilfe eine Leitlinie entwickelt werden kann, auch wenn die wissenschaftliche Grundlage schwach ist. Mit diesem Verfahren könnten Leitlinien auch in anderen Bereichen der Kieferorthopädie mit mangelhafter Evidenz entworfen werden, von denen es wahrlich viele gibt [19]. Die Leitlinie und ihre Empfehlungen beruhen auf wissenschaftlicher Evidenz, sie sind daher direkt auf andere Länder übertragbar. Unterschiede zwischen Gesundheitssystemen, Versorgungsstrukturen und Krankenversicherungen sowie kulturelle Unterschiede können es jedoch rechtfertigen, dass die Empfehlungen einer Leitlinie von Land zu Land unterschiedlich ausfallen, obwohl deren wissenschaftliche Grundlage unverändert bleibt [20]. So wird bspw. in der aktuellen Leitlinie die Verwendung einer elektrischen Zahnbürste empfohlen, obwohl es keinen Nachweis darüber gibt, dass eine elektrische Zahnbürste einer Handzahnbürste bei der Kariesprophylaxe überlegen ist. Die Expertenkommission war der Ansicht, diese Empfehlung wäre für die Niederlande von Bedeutung, da sie der Überzeugung war, Plaque lasse sich mit einer elektrischen Zahnbürste effektiver und einfacher entfernen.

Die vorliegende Leitlinie kann ungeachtet geografischer Unterschiede als Grundlage für die Entwicklung neuer nationaler Leitlinien dienen, da die Ausgangssituation, also risikobehaftete Entscheidungen, überall vorhanden ist. Die Bedeutung einer Leitlinie zur Kariesprophylaxe und die Bestimmung ihrer Effektivität im Rahmen einer praxisorientierten Forschung liegt darin, eine Brücke zwischen Wissenschaft und klinischer Praxis zu schlagen [3, 21]. Ist eine Leitlinie erst einmal erfolgreich implementiert, so kann sie als Goldstandard dienen, mit dem die Effektivität neuer viel versprechender Prophylaxemaßnahmen und die Bereitschaft von Praktikern verglichen werden kann, sich zu verändern und neue Verfahren anzunehmen. Das hier vorgestellte Verfahren ist also eine wirkungsvolle Möglichkeit, die Qualität der Patientenversorgung in der täglichen Praxis zu verbessern. 
- Tab. 5 Zusammenstellung der in der Leitlinie ( Abb. 4) verwendeten Abkürzungen.

Allgemeine prophylaktische Maßnahmen/Empfehlungen (general preventive measures - GM) vor einer Behandlung mit

1. Die Mundhöhle sollte vor Beginn der Behandlung frei von Plaque, Karies und Gingivitis sein

2. Erläuterung des aktuellen oralen Gesundheitszustandes

3. Anweisungen für eine zahnschonende Ernährung und Mundhygiene

4. Mundhygieneunterweisung, Beurteilung der psychomotorischen Fähigkeiten und, falls nicht ausreichend, Anweisung zur Verwendung einer elektrischen Zahnbürste

5. Applikation von Fluoridlack auf sichtbare/vorhandene WSL

6. Zähneputzen 2-mal täglich mit hoch-fluoridhaltiger Zahnpasta (1450 ppm Fluorid)

Beginn der Behandlung (start treatment - ST) bei ausreichender oraler Gesundheit

Verschieben der Behandlung (postpone treatment - PT) bis spezielle Anforderungen der allgemeinen Maßnahmen/Empfehlungen erfüllt sind, wie etwa ausreichend gute Mundhygiene und geringere Kariesanfälligkeit durch geringeren Zuckerkonsum

Spezielle prophylaktische Maßnahmen/Empfehlungen während einer Behandlung mit festsitzenden Apparaturen

Verwendung einer fluoridhaltigen Zahnpasta (1450 ppm Fluorid)

Entfernen der Drähte, Reinigung/Politur der Zähne und Applikation von Fluoridlack (fluoride varnish - FV)

Anweisungen zur Ernährung (dietary advice-DA) und zur Mundhygiene

schriftliche Benachrichtigung (der Eltern) des Patienten mit Erläuterung des Problems und Kopie an den Allgemeinzahnarzt (Patientenbrief 1)

schriftliche Benachrichtigung (der Eltern) des Patienten mit dem Hinweis, dass Initialkaries erkennbar ist mit Erläuterung des Problems und

Kopie an den Allgemeinzahnarzt (Patientenbrief 2)

schriftliche Benachrichtigung der (Eltern des) Patienten über die Entbänderung (Entfernung sämtlicher festsitzender Apparaturen) mit

Kopie an den Allgemeinzahnarzt (Patientenbrief 3)

Entbänderung (Debonding - DB - Entfernung sämtlicher festsitzender Apparaturen ohne Einsetzen eines Dauerretainers)

\section{Klinische Relevanz}

White-Spot-Läsionen sind in der Kieferorthopädie ein großes Problem. Evidenzbasierte Leitlinien für die Prophylaxe liegen noch nicht vor. Daher kann die vorgelegte neu entwickelte Leitlinie beim Umgang mit diesem Phänomen hilfreich sein.

\section{Danksagungen}

Die Autoren danken den Mitgliedern der Expertenkommission für ihre Unterstützung und fachliche Beratung, $\mathrm{H}$. Hoogenboom für seine Hilfe bei der Entwicklung der computergestützten Patientenkarten, E.M. Bronkhorst für das Datenmanagement und IV Dirks, H. van Diemen, A. Derks für ihre Beiträge zur Studie.

\section{Literatur}

[1] Julien KC, Buschang PH, Campbell PM. Prevalence of white spot lesion formation during orthodontic treatment. Angle Orthod 2013; 83: 641 $-647$

[2] Ogaard B, Rølla G, Arends J et al. Orthodontic appliances and enamel demineralization. Part 2. Prevention and treatment of lesions. Am J Orthod Dentofacial Orthop 1988; 94: $123-128$

[3] Derks A, Kuijpers-Jagtman AM, Frencken JE et al. Caries preventive measures used in orthodontic practices: an evidence-based decision? Am J Orthod Dentofacial Orthop 2007; 132: 165 -170
[4] Derks A, Katsaros C, Frencken JE et al. Caries-inhibiting effect of preventive measures during orthodontic treatment with fixed appliances. A systematic review. Caries Res 2004; 38: $413-420$

[5] Benson PE, Parkin N, Dyer F et al. Fluorides for the prevention of early tooth decay (demineralised white lesions) during fixed brace treatment. Cochrane Database Syst Rev 2013; 12: CD003809

[6] van der Sanden W], Mettes DG, Plasschaert AJ et al. Clinical practice guidelines in dentistry: opinions of dental practitioners on their contribution to the quality of dental care. Qual Saf Health Care 2003; 12: $107-111$

[7] Keeney S, Hasson F, McKenna HP. A critical review of the Delphi technique as a research methodology for nursing. Int J Nurs Stud 2001; 38: $195-200$

[8] Stecksén-Blicks C, Renfors G, Oscarson ND et al. Caries-preventive effectiveness of a fluoride varnish: a randomized controlled trial in adolescents with fixed orthodontic appliances. Caries Res 2007; 41: $455-459$

[9] Benson PE, Shah AA, Millett DT et al. Fluorides, orthodontics and demineralization: a systematic review. J Orthod 2005; 32: $102-114$

[10] Benson PE, Parkin N, Millett DT et al. Fluorides for the prevention of white spots on teeth during fixed brace treatment. Cochrane Database Syst Rev 2004; 3: CD003809

[11] Chadwick BL, Roy J, Knox J et al. The effect of topical fluorides on decalcification in patients with fixed orthodontic appliances: a systematic review. Am J Orthod Dentofacial Orthop 2005; 128: 601 $-606$

[12] Zimmer BW, Rottwinkel Y. Assessing patient-specific decalcification risk in fixed orthodontic treatment and its impact on prophylactic procedures. Am J Orthod Dentofacial Orthop 2004; 126: 318 -324 
[13] Brouwers MC, Kho ME, Browman GP et al.AGREE Next Steps Consortium. AGREE II: advancing guideline development, reporting and evaluation in health care. J Clin Epidemiol 2010; 63: 1308 -1311

[14] Fitch K, Bernstein S], Aguilar MD et al. The RAND/UCLA Appropriateness Method User's Manual. Santa Monica, CA: RAND Corp.; 2001

[15] Jones ], Hunter D. Consensus methods for medical and health services research. BMJ 1995; 311: $376-380$

[16] Jiang $\mathrm{H}$, Hua F, Yao L et al. Effect of $1.23 \%$ acidulated phosphate fluoride foam on white spot lesions in orthodontic patients: a randomized trial. Pediatr Dent 2013; 35: 275 -278

[17] Robertson MA, Kau CH, English JD et al. MI Paste Plus to prevent demineralization in orthodontic patients: a prospective randomized controlled trial. Am J Orthod Dentofacial Orthop 2011; 140: 660 -668
[18] O’Reilly MT, De Jesús Viñas J, Hatch JP. Effectiveness of a sealant compared with no sealant in preventing enamel demineralization in patients with fixed orthodontic appliances: a prospective clinical trial. Am J Orthod Dentofacial Orthop 2013; 143: 837 -844

[19] O'Brien K, Sandler J. In the land of no evidence, is the salesman king? Am J Orthod Dentofacial Orthop 2010; 138: 247 -249

[20] Christiaens T, De Backer D, Burgers J et al. Guidelines, evidence, and cultural factors. Scand J Prim Health Care 2004; 22: 141 -145

[21] Westfall JM, Mold J, Fagnan L. Practice-based research - "Blue Highways" on the NIH roadmap. JAMA 2007; 297: $403-406$ 